\title{
Função polinomial do segundo grau: uma prática utilizando o software geogebra
}

\author{
Second degree polynomial function: a practice using geogebra software \\ Jefferson Dantas de Oliveira ${ }^{1}$ \\ Zulma Elizabete de Freitas Madruga ${ }^{2}$
}

Resumo :Este artigo apresenta um relato de experiência de estágio que consistiu na aplicação de atividades envolvendo o software Geogebra em uma turma de $1^{\circ}$ ano do Ensino Médio de uma escola pública do sul da Bahia. A prática objetivou desenvolver com os estudantes uma atividade investigativa de função polinomial do segundo grau. A realização se deu durante o horário de aula, por meio de uma oficina, onde os estudantes apresentaram para os colegas os gráficos elaborados no software. Ao final da oficina os estudantes manifestaram-se positivamente, dizendo que o software proporcionou uma melhor visualização e entendimento da representação gráfica das funções do $2^{\circ}$ grau estudadas.

Palavras-chave: Ensino de Matemática. Função do $2^{\circ}$ grau. Geogebra.

Abstract:This article presents an experience report that consisted in the application of activities involving the Geogebra software in a class of 1st year of the High School of a public school in the south of Bahia. The practice aimed to develop with the students a research activity of polynomial function of the second degree. The realization took place during the class hours, through a workshop, where the students presented to the colleagues the graphics elaborated in the software. At the end of the workshop, the students expressed a positive opinion, saying that the software provided a better visualization and understanding of the graphical representation of the secondary functions studied.

Keywords: Mathematics Teaching. Function of the second degree. Geogebra.

\section{Introdução}

\footnotetext{
${ }^{1}$ Mestrando em Educação Matemática UESC. Licenciado em Matemática. E-mail: dantascpm2a@hotmail.com

${ }^{2}$ Doutora em Educação em Ciências e Matemática. Docente do Programa de Pós-graduação em Educação Matemática - PPGEM/UESC E-mail: betefreitas.m@gmail.com
}

Tangram - Revista de Educação Matemática, Dourados - MS - v.1 n. 1, pp. 102 - 113 (2018) 


\section{Função polinomial do segundo grau: uma prática utilizando o software geogebra}

Um dos requisitos básico para conclusão do curso de graduação em Licenciatura em Matemática é a realização do Estágio Supervisionado. Esta disciplina é fragmentada em quatro partes, nas quais os graduandos devem participar de atividades em escolas da Educação Básica para observar e aplicar na prática os conteúdos estudados teoricamente.

Enquanto cursava o $9^{\circ}$ semestre do curso de Matemática, o primeiro autor deste relato realizou estágio no Colégio da Polícia Militar (CPM) no município de Itabuna/BA. O início deste estágio ocorreu em maio de 2016 no turno matutino em uma turma do $1^{\circ}$ ano do Ensino Médio, com a supervisão da professora da disciplina.

O Colégio da Polícia Militar (CPM) de Itabuna/BA foi fundado em 2005, com o intuito de melhorar a qualidade de ensino das escolas públicas na cidade, esta intenção está explícita no regimento escolar. O colégio tem uma excelente estrutura; todas as salas de aula são equipadas com ar condicionado, quadro branco, além de uma televisão pendrive, uma realidade distante das outras escolas públicas da cidade, que possuem ainda algumas dificuldades relacionadas às questões estruturais.

$\mathrm{Na}$ parte pedagógica, o CPM conta um quadro de professores com formação em nível de mestrado e doutorado. Como a visão da escola é instigar o estudante para superar seus limites, os professores da escola têm a missão de desafiá-los constantemente. Na turma onde ocorreu o estágio, a disciplina Matemática possuía carga horária de quatro horas aulas semanais.

Esse estágio consistia em observar as aulas de Matemática na referida turma durante 15 horas aulas, e em seguida aplicar uma atividade com o objetivo de trabalhar de forma lúdica os conteúdos de Matemática abordados pela professora.

Durante o estágio, o conteúdo trabalhado foi funções polinomiais do segundo grau, na qual a professora inicialmente explorava os coeficientes $a, b$ e $c$, de uma função algébrica, em seguida começou a trabalhar com as raízes de uma equação, eixo de simetria e o desenho do gráfico em um ambiente papel e lápis. No entanto, percebeu-se a dificuldade dos estudantes em entender alguns conceitos relacionados a este conteúdo apenas no ambiente papel e lápis.

Com base nestas observações, teve-se a ideia de trazer este mesmo conteúdo no ambiente computacional, surgindo assim o seguinte questionamento: o software Geogebra pode auxiliar na aprendizagem dos estudantes do conteúdo de função polinomial do

Tangram - Revista de Educação Matemática, Dourados - MS - v.1 n. 1, pp. 102 - 113 (2018) 


\section{Função polinomial do segundo grau: uma prática utilizando o software geogebra}

segundo grau? Para responder a esta questão, objetivou-se desenvolver com os estudantes uma atividade investigativa de função polinomial do segundo grau, com o auxilio do software Geogebra.

\section{Fundamentação Teórica}

O avanço tecnológico decorrido nas últimas décadas tem gerado aparelhos sofisticados com múltiplas funções e maior desempenho nas atividades que desenvolvem, tais como: celulares, notebooks, tablets, smartphones, televisão, entre outros.

Este avanço das tecnologias atrai atenção e interesse de grande parte dos estudantes que frequentam os colégios da Educação Básica. Muitas vezes é possível observar os estudantes em sala de aula com smartphone ou outros aparelhos tecnológicos. Segundo Benitti (2010), os estudantes estão imersos em um ambiente no qual está rodeado de aparelhos tecnologia e a maioria deles não entendem o seu funcionamento.

No cotidiano da sala de aula, é apresentado aos estudantes conceitos e teorias matemáticas por meio do exercício, que parecem estar distante da realidade, porém, eles não têm conhecimento de que a maioria dos conteúdos ali abordados está diretamente ligada com os aparelhos tecnológicos que utilizam.

No livro "A Máquina das Crianças Repensando a Escola na Era da Informática”, Papert (2002), aborda inicialmente o problema de que a tecnologia está presente ao redor das pessoas, assim como em todas as profissões. No entanto, na educação a tecnologia está distante da realidade das salas de aula, e a forma na qual os conteúdos são ensinados para os estudantes continua a mesma. "A escola é um notável exemplo de uma área que não mudou tanto. Pode-se dizer que não houve qualquer mudança na maneira como nós distribuímos a educação aos nossos estudantes" (Papert, 2002, p.10).

Com o uso da tecnologia na sala de aula, o professor dá oportunidade aos estudantes de construir o seu próprio conhecimento, pois, de acordo com a proposta de Brousseau (1975), o estudante em interação com os outros colegas, tem a oportunidade de expor suas ideias e discuti-las com a intenção de validá-las, ou seja, nessa interação o conhecimento é construído pelos estudantes e não necessariamente exposto pelo professor.

Para Júnior (2010) o uso da tecnologia digital na escola é uma maneira de justificar as "falhas" deixadas pela visão tecnicista de ensino, devido ao entusiasmo de responder as

Tangram - Revista de Educação Matemática, Dourados - MS - v.1 n. 1, pp. 102 - 113 (2018) 


\section{Função polinomial do segundo grau: uma prática utilizando o software geogebra}

indagações dos estudantes, acerca da utilidade dos diversos conteúdos abordados na sala de aula e a sua relação com o cotidiano.

$\mathrm{Na}$ busca por uma melhor relação entre o ensino e a aprendizagem em sala de aula, Cowper (1994) afirma que os softwares dão uma nova visão a estes processos, pois aproxima a relação entre professor e estudante, baseando-se na busca pelo conhecimento e tornando-os mais atrativos para o estudante.

Conforme Zulatto (2002), o processo de construção do conhecimento por meio da utilização de softwares para o ensino, possibilita ao estudante o conhecimento por meio da investigação no qual ele pode formular hipóteses, a fim de validá-las ou não. Na busca em tornar a aprendizagem mais atrativa, busca-se propor atividades investigativas nas quais apresentem relações com os conteúdos estudados, proporcionando assim que este estudante seja parte do processo de aprendizagem.

Diante disso percebe-se que a utilização de tecnologias pode facilitar o processo de aprendizagem da Matemática. É importante para estabelecer relação entre a teoria estudada na sala de aula com a prática e o cotidiano dos estudantes.

\section{A observação}

O início do estágio no CPM ocorreu no mês de maio de 2016 no período da manhã em uma turma de $1^{\circ}$ ano do Ensino Médio. Nas primeiras duas semanas ocorreram as observações das aulas ministradas pela professora titular da disciplina. Quando se iniciaram as essas observações, a professora da turma estava abordando conteúdos referentes à função polinomial do $1^{\circ}$ grau. Durante esse período ela abordou sobre coeficientes (angular e linear), translação do gráfico, obtenção a lei da função por meio do gráfico, e depois explanação sobre os conceitos, propunha atividades para que os estudantes pudessem fixar o conteúdo.

A partir desse dia, a cada aula, a professora adiantava os conteúdos da disciplina, já que no CPM os prazos das unidades são curtos e não há muito tempo para revisões. Na aula seguinte a professora levou para sala papel quadriculado, para trabalhar a construção de um gráfico de uma função polinomial do $1^{\circ}$ grau. Nas aulas seguintes, ela explanou sobre estudo do sinal de uma função e no final dessa mesma aula já marcou o primeiro teste de classe da unidade para a próxima aula, encerrando assim o conteúdo de função polinomial do $1^{\circ}$ grau.

Tangram - Revista de Educação Matemática, Dourados - MS - v.1 n. 1, pp. 102 - 113 (2018) 


\section{Função polinomial do segundo grau: uma prática utilizando o software geogebra}

Na aula seguinte após o teste, a professora iniciou o conteúdo de função polinomial do $2^{\circ}$ grau no qual começou a trabalhar os conceitos de coeficientes, e suas relações com o gráfico da função. No final dessa aula o colégio entrou em recesso para os festejos juninos.

Após o retorno das aulas, a professora deu continuidade ao conteúdo explanando sobre zeros da função e vértice. Depois do conteúdo dado, ela passou exercícios para que os estudantes pudessem praticar o que tinha sido aprendido.

$\mathrm{Na}$ aula seguinte a professora iniciou a parte final do conteúdo englobando os assuntos de estudo do sinal e eixo de simetria, vale salientar que nesta aula os estudantes tiveram bastante dificuldade para entender o conteúdo. Então, nesse dia foi avisado que na próxima aula o estagiário assumiria a turma a fim de realizar uma atividade de interação para desenvolver o estudo de funções.

Assim, o primeiro autor deste relato apresentou-se a turma, e explicou que aplicaria uma oficina, e como culminância, os estudantes iriam apresentar no software Geogebra os conteúdos aprendidos em sala referentes à função polinomial do segundo grau.

\section{A Oficina}

No planejamento do estágio ocorreu uma discussão com a professora da disciplina sobre o que poderia ser feito em relação a oficina. A professora deu inicialmente a ideia de se trabalhar com uma atividade em forma de lista para ser realizada em grupo, mas no ambiente papel e lápis. Porém, depois ela teve a ideia de se utilizar um software de computação, então sugerir a professora os softwares Geogebra e Winplot.

A professora já tinha ouvido falar do software Geogebra, então se decidiu pela utilização do mesmo para se trabalhar o conteúdo de funções do segundo grau, pois além de facilitar a aprendizagem, poderia despertar por meio deste artefato tecnológico, maior interesse por parte dos estudantes.

Então na segunda semana de julho de 2016 iniciou-se o estágio propriamente dito. Nesta oportunidade foi apresentado aos estudantes o software Geogebra, todas as suas funções e os componentes da sua interface gráfica. Nesta aula, utilizou-se o conteúdo de função polinomial do $2^{\circ}$ grau, para trabalhar as funções dadas pela professora e construir o gráfico, marcando os pontos dos coeficientes $a, b$ e $c$; zeros da função, vértice e eixo de simetria, além do estudo do sinal.

Tangram - Revista de Educação Matemática, Dourados - MS - v.1 n. 1, pp. 102 - 113 (2018) 


\section{Função polinomial do segundo grau: uma prática utilizando o software geogebra}

Com a utilização do software para uma segunda abordagem do conteúdo, os estudantes conseguiram compreender graficamente o que estava sendo calculado algebricamente. Então se dedicou o tempo da aula para realização de exemplos. Nesses exemplos, foram inseridas diversas funções polinomiais do segundo grau no software, e em seguida, foi destacado através do gráfico da função, as implicações dos coeficientes a, b e c; a localização das raízes no gráfico; a posição do vértice da parábola e o eixo de simetria correspondente ao gráfico da função. Vale salientar que todas essas aplicações foram repetidas em vários exemplos, a fim de que os estudantes conseguissem visualizar graficamente os cálculos produzidos algebricamente.

No final da aula foi proposta aos estudantes a realização de uma atividade: foi entregue uma folha com cinco funções, com a finalidade que eles se organizassem em cinco grupos, desenvolvessem todas as aplicações das funções vistas em sala de aula no software, e ainda apresentassem uma das questões na semana seguinte, que seria escolhida através de um sorteio.

$\mathrm{Na}$ aula seguinte, iniciou-se com o recebimento dos trabalhos realizados pelos estudantes. Esse trabalho, escrito, foi solicitado para que expressassem os cálculos algébricos e os gráficos solicitados na aula anterior. Após a entrega da atividade escrita, foi realizado um sorteio, no qual foi selecionada uma questão da lista de exercício para cada grupo. Durante a apresentação, os estudantes inseriam a função determinada no software, e após o Geogebra apresentar o gráfico correspondente, eles com os auxilio das ferramentas disponíveis no programa evidenciavam: 1) as implicações dos coeficientes a, b e c sofridas pelo gráfico da função, 2) os zeros da função, 3) a posição do vértice da parábola e 4) o eixo de simetria. Em seguida, será feita uma análise da função $f(x)=x^{2}-5 . x+6$ correspondente à atividade apresentada pelos estudantes.

\section{1) Implicações dos coeficientes a, b e c.}

Segundo Souza (2013), a função polinomial do segundo grau completa, é descrita algebricamente da seguinte foma: $f(x)=a \cdot x^{2}+b \cdot x+c$, no qual os termos $a, b$ e c são números reais com a diferente de zero, pois se o coeficiente a for igual a zero a função automaticamente é denominada polinomial do primeiro grau. Segundo o autor o termo a, corresponde graficamente a concavidade da parábola, se o termo a for maior que zero, então a concavidade da mesma é voltada para cima, caso o a seja menor que zero a concavidade é voltada para baixo. O termo b, graficamente está associado a curva que

Tangram - Revista de Educação Matemática, Dourados - MS - v.1 n. 1, pp. 102 - 113 (2018) 


\section{Função polinomial do segundo grau: uma prática utilizando o software geogebra}

intercepta o eixo y, se a curva interceptar o eixo y na parte crescente então o coeficiente b é maior que zero, caso ele intercepte o termo b na parte decrescente então o termo b é menor que zero, e se o gráfico foi interceptado entre as curvas decrescente e crescente, então o b é igual a zero. O termo c, corresponde graficamente o ponto que intercepta o eixo y, este ponto algebricamente é descrito por $(0, \mathrm{c})$. Na figura 1 , tem-se a analise dos coeficientes correspondentes ao gráfico da função $f(x)=x^{2}-5 \cdot x+6$.

Figura 1: Análise dos coeficientes da Função f.

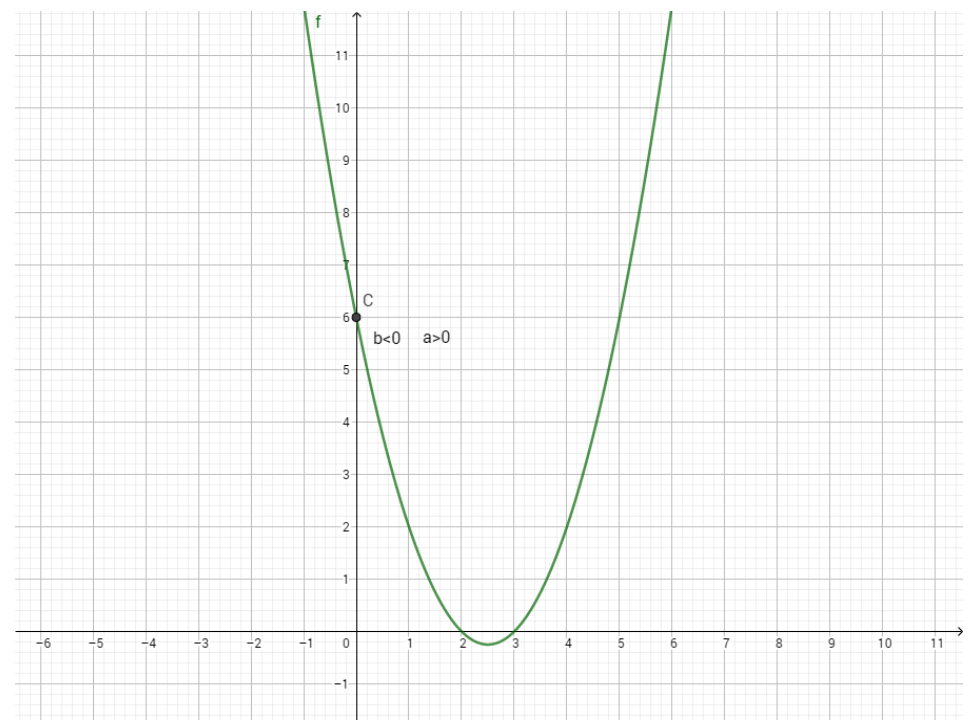

Fonte: Elaborado pelos autores (2018)

Conforme pode ser visualizado na figura 1 , o termo a é maior que zero, já que a parábola tem concavidade para cima. $\mathrm{O}$ termo $\mathrm{b}$, é menor que zero, pois observe que a parábola intercepta o eixo y, na parte decrescente da curva, e o termo c é igual a 6 , pois observa-se que o ponto que intercepta o eixo y é $(0,6)$.

$\mathrm{Na}$ realização da oficina, essas eram as informações que foram solicitadas aos estudantes como tarefa 1, a ser explorada durante a explicação do gráfico produzido no Geogebra. Vale salientar que nesta parte da atividade os estudantes não apresentaram muitas dificuldades para realiza-la.

\section{2) Os zeros da função.}

Conforme Souza (2013), os zeros da função correspondem a todo valor de $x$ pertencente ao seu domínio, tal que $\mathrm{f}(\mathrm{x})=0$, ou seja, é todo valor atribuído a $\mathrm{x}$ de forma que quando aplicado na função, o valor de $y=0$. Uma função polinomial do segundo grau algebricamente pode possuir: duas raízes reais distintas, caso delta for maior que zero, duas

Tangram - Revista de Educação Matemática, Dourados - MS - v.1 n. 1, pp. 102 - 113 (2018) 


\section{Função polinomial do segundo grau: uma prática utilizando o software geogebra}

raízes reais iguais, caso o delta seja igual a zero, e não possuir nenhuma raiz real, caso o delta seja menor que zero.

A abordagem gráfica de uma função polinomial do segundo grau, segundo os estudantes que participaram da oficina, é mais fácil de compreender do que a abordagem algébrica, pois visualizando o gráfico da função é intuitivo observar se ele interceptou o eixo $\mathrm{x}$, em um ponto, em dois pontos ou em nenhum ponto. Dessa forma, ao interceptar o eixo x em algum ponto, significa que o valor para y neste ponto é igual a zero, em conformidade com a definiçãa algébrica apresentada anteriormente. Na figura 2, tem-se a análise gráfica das raízes da função $f(x)=x^{2}-5 \cdot x+6$.

Figura 2: Análise das raízes da função f

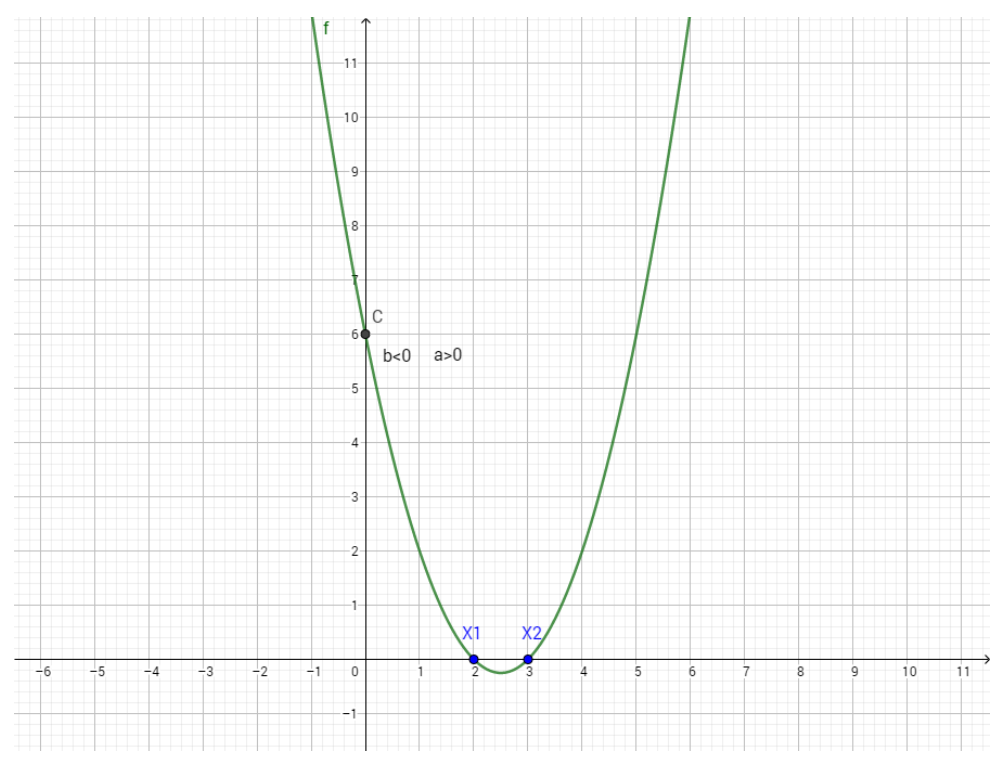

Fonte: Elaborado pelos autores (2018)

Conforme pode ser visualizado na figura 2 , o gráfico da função f, intercepta o eixo $\mathrm{x}$, apenas em dois pontos o x1, e x2, dessa forma pode-se concluir que a função apresenta raízes iguais a $X_{1}=2$ e $X_{2}=3$, concluindo-se também que o delta é maior que zero.

\section{3) Posição do vértice da parábola}

De acordo com Souza (2013), o vértice de uma parábola, algebricamente, é encontrado através da equação: $\mathrm{V}=(\mathrm{Xv}=-\mathrm{b} / 2 . \mathrm{a} ; \mathrm{Yv}=-\Delta / 4 . \mathrm{a})$. A abordagem gráfica por sua vez, é apresentada pelo autor de forma mais simplória, segundo o mesmo, graficamente o vértice corresponde ao ponto central da parábola, ou seja, o ponto que separa as duas curvas simétricas da parábola, e esse ponto é classificado como: ponto de mínimo e de máximo; o

Tangram - Revista de Educação Matemática, Dourados - MS - v.1 n. 1, pp. 102 - 113 (2018) 
ponto de mínimo é encontrado quando o termo a é maior que zero, e o ponto de máximo é encontrado quando o termo a é menor que zero.

Na figura 3, apresenta-se a localização do vértice da parábola correspondente ao gráfico da função $f(x)=x^{2}-5 \cdot x+6$.

Figura 3: Análise do Vértice da função f.

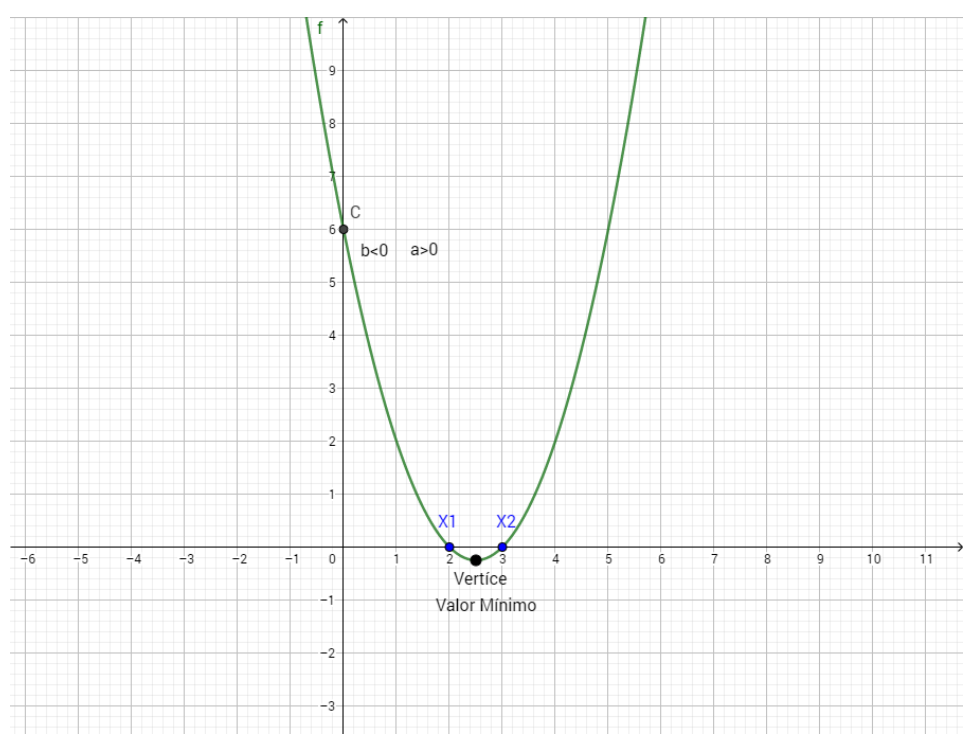

Fonte: Elaborado pelos autores (2018)

\section{4) Eixo de simetria}

O eixo de simetria de uma parábola segundo Souza (2013), o intercepta em seu vértice, dessa forma para determinar o eixo de simetria de uma parábola, deve ser determinado primeiro o vértice, e em seguida é traçada a reta que o intercepta.

Os estudantes nas aulas da disciplina tinham grande dificuldade em entender como era encontrada a reta que passa pelo vértice, uma vez que, os mesmos apresentavam dificuldades para o encontrar. Na oficina, foi praticado diversas vezes, a forma de encontrar o vértice e o eixo de simetria no software Geogebra. Na figura 4, tem-se o eixo de simetria da função $f$.

Figura 4: Eixo de Simetria da função f.

Tangram - Revista de Educação Matemática, Dourados - MS - v.1 n. 1, pp. 102 - 113 (2018) 


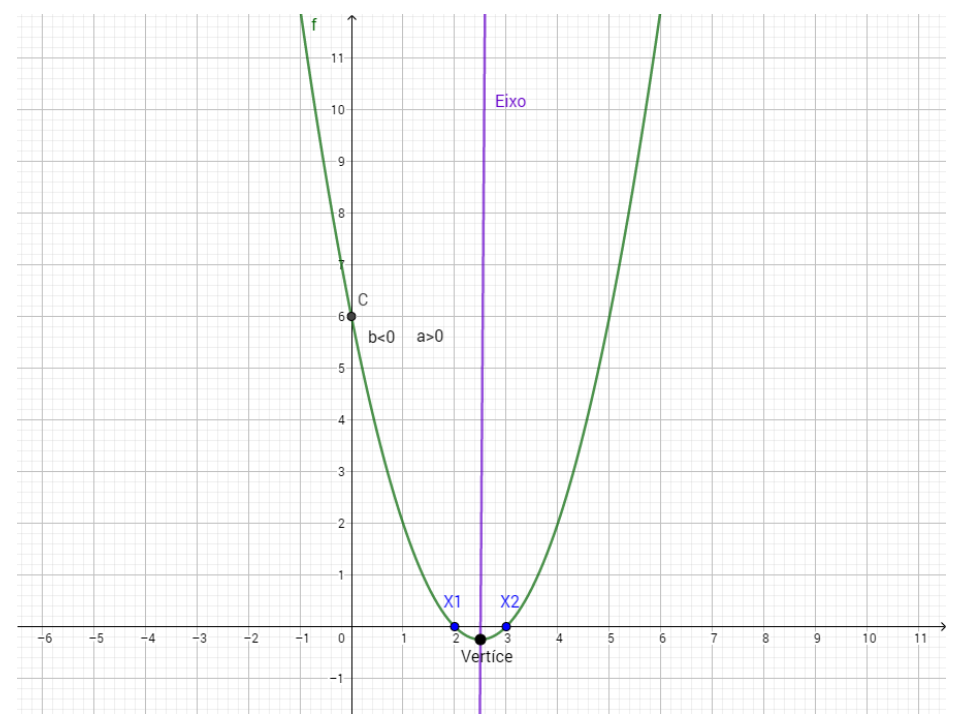

Fonte: Elaborado pelos autores (2018)

No software Geogebra, para determinar o eixo de simetria, é intuitivo para os alunos, de forma que, já que haviam encontrado o vértice na atividade 3 , nesta atividade apenas precisam selecionar nas ferramentas disponíveis no programa, uma reta e em seguida, liga-la com o vértice e um outro ponto qualquer que configure uma reta.

Com a prática que desenvolveram no software, os participantes da oficina adquiriram confiança no momento de determinar o vértice e o eixo de simetria de uma função, dificuldade comumente apresentada antes da realização desta oficina. Nas figuras 5 e 6 apresenta-se alguns alunos durante a exposição da oficina.

Figura 5 - Aluno 1 manipulando o software

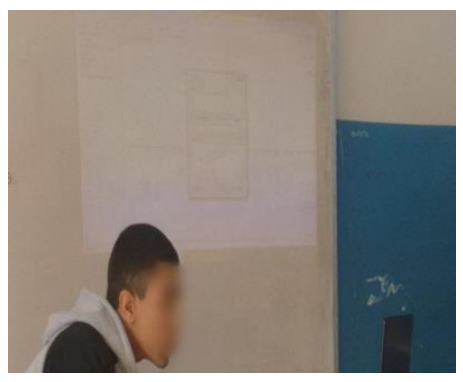

Fonte: Os autores (2018)
Figura 6 - Aluno 2 manipulando o software

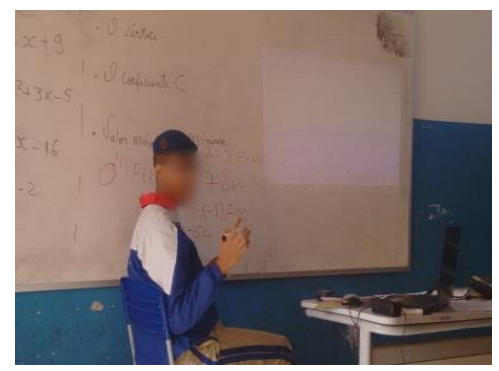

Fonte: Os autores (2018)

\section{Considerações Finais}

$\mathrm{Na}$ aplicação da oficina pode-se observar que a utilização do software Geogebra para o ensino de funções polinomiais do segundo grau, auxiliou na aprendizagem do conteúdo 


\section{Função polinomial do segundo grau: uma prática utilizando o software geogebra}

em questão. A manipulação e apresentação do software perante os colegas fez com que diversos estudantes fossem buscar o conteúdo em livros e outros meios de estudo, a fim que conseguissem apresentar para os demais colegas, isto mostra que eles fizeram parte do processo de ensino e aprendizagem, conforme afirma Papert (2002).

Vale salientar que este estágio foi importante para aquisição de experiência em sala de aula como professor. A regência com supervisão oportuniza a aprendizagem do futuro professor, tanto com a prática, com o professor da disciplina, e com os próprios estudantes.

No final das apresentações foi questionado aos estudantes o que eles acharam da utilização do software no ensino e aprendizagem de função quadrática. Alguns dos estudantes que se pronunciaram disseram que foi de grande importância para a visualização da parábola; outros disseram que ficou mais fácil de entender o que significa as raízes de uma função e vértice; outros falaram que a visualização no software facilitou a aprendizagem. Porém um estudante que mencionou um ponto como negativo: ele disse que o software não tem uma interface muito simples para ser trabalhada e precisa de um tutorial para poder manuseá-lo com mais facilidade. Esses tutoriais existem, no entanto, não foi utilizado na turma, pois se pensou que somente a apresentação inicial do estagiário seria o suficiente.

Conforme a avaliação positiva apresentada pelos estudantes pode-se inferir que a utilização de um software com o objetivo de dinamizar o processo de ensino e aprendizagem da Matemática, é de grande relevância para os alunos e para os professores, conforme havia previsto Papert (2002).

\section{Referências}

Benitti, Fabiane. (2012) Exploring the Educational Potential of Robotics in Schools: A Systematic Review. Universidade do Vale do Itajaí (UNIVALI), CTTMar, Programa de Pós-Graduação em Computação Aplicada, Rua Uruguai, 458, CP 360, 88302-202 Itajaí, SC, Brazil.

Brousseau, G. (1986) Fondements et méthodes de la didactique des mathématiques. Recherche en didactique des mathématiques, Grenoble, v. 7, n. 2, p. 33-115.

Cowper, W. (1994) Exploring drag-mode geometry. In: Discovering Geometry with a computer - Using Cabri Géomètre. Heinz Schumann and David Green. Ed. ChartwellBratt, 1994.

Tangram - Revista de Educação Matemática, Dourados - MS - v.1 n. 1, pp. 102 - 113 (2018) 
Função polinomial do segundo grau: uma prática utilizando o software geogebra

Junior, Raul. O Ensino a distância e as novas tecnologias. Revista Primus Vitam-N${ }^{\circ}$. 2013.

Papert, S. A Máquina das Crianças: Repensando a Escola na Era da Informática. Porto Alegre: Artes Médicas, 2002.

Souza, J. R. Novo olhar: Matemática : 1 / Joamir Roberto de Sousa. - 2. Ed. - São Paulo: FTD, 2013.

Zulatto, R. B. A. Professores de Matemática que utilizam softwares de Geometria Dinâmica: suas características e perspectivas. Dissertação de Mestrado. Instituto de Geociências e Ciências Exatas, Universidade Estadual Paulista, Rio Claro, 2002.

Recebido: $11 / 01 / 2018$

Aceito: 04/03/2018

Tangram - Revista de Educação Matemática, Dourados - MS - v.1 n. 1, pp. 102 - 113 (2018) 